\title{
Predicting Salient Updates for Disaster Summarization
}

\author{
Chris Kedzie and Kathleen McKeown \\ Columbia University \\ Department of Computer Science \\ \{kedzie, kathy\}@cs.columbia.edu
}

\author{
Fernando Diaz \\ Microsoft Research \\ fdiaz@microsoft.com
}

\begin{abstract}
During crises such as natural disasters or other human tragedies, information needs of both civilians and responders often require urgent, specialized treatment. Monitoring and summarizing a text stream during such an event remains a difficult problem. We present a system for update summarization which predicts the salience of sentences with respect to an event and then uses these predictions to directly bias a clustering algorithm for sentence selection, increasing the quality of the updates. We use novel, disaster-specific features for salience prediction, including geo-locations and language models representing the language of disaster. Our evaluation on a standard set of retrospective events using ROUGE shows that salience prediction provides a significant improvement over other approaches.
\end{abstract}

\section{Introduction}

During crises, information is critical for first responders, crisis management organizations, and those caught in the event. When the event is significant, as in the case of Hurricane Sandy, the amount of content produced by traditional news outlets, government agencies, relief organizations, and social media can vastly overwhelm those trying to monitor the situation. Crisis informatics (Palen et al., 2010) is dedicated to finding methods for sharing the right information in a timely fashion during such an event. Research in this field has focused on human-in-the-loop approaches ranging from on the ground information gathering to crowdsourced reporting and disaster management (Starbird and Palen, 2013).

Multi-document summarization has the potential to assist the crisis informatics community. Automatic summarization could deliver relevant and salient information at regular intervals, even when human volunteers are unable to. Perhaps more importantly it could help filter out unnecessary and irrelevant detail when the volume of incoming information is large. While methods for identifying, tracking, and summarizing events from text based input have been explored extensively (Allan et al., 1998; Filatova and Hatzivassiloglou, 2004; Wang et al., 2011), these experiments were not developed to handle streaming data from a heterogeneous environment at web scale. These methods also rely heavily on redundancy which is suboptimal for time sensitive domains where there is a high cost in delaying information.

In this paper, we present an update summarization system to track events across time. Our system predicts sentence salience in the context of a large-scale event, such as a disaster, and integrates these predictions into a clustering based multidocument summarization system. We demonstrate that combining salience with clustering produces more relevant summaries compared to baselines using clustering or relevance alone. Our experiments suggest that this is because our system is better able to adapt to dynamic changes in input volume that adversely affect methods that use redundancy as a proxy for salience.

In addition to the tight integration between clustering and salience prediction, our approach also exploits knowledge about the event to determine salience. Thus, salience represents both how typical a sentence is of the event type (e.g., industrial accident, hurricane, riot) and whether it specifies information about this particular event. Our feature representation includes a set of language models, one for each event type, to measure the typicality of the sentence with regard to the current event, the distance of mentioned locations from the center of the event, and the change in word frequencies over the time of the event. While we evaluate these features in the domain of disasters, 
this approach is generally applicable to many update summarization tasks.

Our approach achieves a statistically significant improvement in ROUGE scores compared to multiple baselines. Additionally, we introduce novel methods for estimating the average information gain each update provides and how completely the update summary covers the event it is tracking; our system's updates contain more relevant information on average than the competing baselines.

The remainder of the paper is organized as follows. We begin with a review of related work in the information retrieval and multi-document summarization literature. Section 3 outlines the details of our salience and summarization models. Next we describe our data (Section 4) and experiments (Section 5). Finally, we discuss our results (Section 6) and conclude the paper.

\section{Related Work}

A principal concern in extractive multi-document summarization is the selection of salient sentences for inclusion in summary output (Nenkova and McKeown, 2012). Existing approaches generally fall into one of three categories, each with specific trade-offs with respect to update summarization.

First, centrality-focused approaches (including graph (Erkan and Radev, 2004), cluster (Hatzivassiloglou et al., 2001), and centroid (Radev et al., 2004) methods) are very natural for retrospective analysis in the sense that they let the data "speak for itself." These methods equate salience with centrality, either to the input or some other aggregate object (i.e. a cluster center or input centroid). However, they rely chiefly on redundancy. When applied to an unfolding event, there may not exist enough redundant content at the event onset for these methods to exploit. Once the event onset has passed, however, the redundancy reduction of these methods is quite beneficial.

The second category, predictive approaches, includes ranking and classification based methods. Sentences have been ranked by the average word probability, average TF*IDF score, and the number of topically related words (topic-signatures in the summarization literature) (Nenkova and Vanderwende, 2005; Hovy and Lin, 1998; Lin and Hovy, 2000). The first two statistics are easily computable from the input sentences, while the third only requires an additional, generic background corpus. In classification based methods, model features are usually derived from human generated summaries, and are non-lexical in nature (e.g., sentence starting position, number of topic-signatures, number of unique words). Seminal work in this area has employed naïve Bayes and logistic regression classifiers to identify sentences for summary inclusion (Kupiec et al., 1995; Conroy et al., 2001). While these methods are less dependent on redundancy, the expressiveness of their features is limited. Our model expands on these basic features to account for geographic, temporal, and language model features.

The last category includes probabilistic (Haghighi and Vanderwende, 2009), information theoretic, and set cover (Lin and Bilmes, 2011) approaches. While these methods are focused on producing diverse summaries, they are difficult to adapt to the streaming setting, where we do not necessarily have a fixed summary length and the corpus to be summarized contains many irrelevant sentences, i.e. there are large portions of the corpora that we specifically want to avoid.

Several researchers have recognized the importance of summarization during natural disasters. (Guo et al., 2013) developed a system for detecting novel, relevant, and comprehensive sentences immediately after a natural disaster. (Wang and $\mathrm{Li}, 2010$ ) present a clustering-based approach to efficiently detect important updates during natural disasters. The algorithm works by hierarchically clustering sentences online, allowing the system to output a more expressive narrative structure than (Guo et al., 2013). Our system attempts to unify these system's approaches (predictive ranking and clustering respectively).

\section{Method}

Our update summarization system takes as input a) a short query defining the event to be tracked (e.g. 'Hurricane Sandy'), b) an event category defining the type of event to be tracked (e.g. 'hurricane'), c) a stream of time-stamped documents presented in temporal order, and $d$ ) an evaluation time period of interest. While processing documents throughout the time period of interest, the system outputs sentences from these documents likely to be useful to the query issuer. We refer to these selected sentences as updates.

In order to measure the usefulness of a system's updates, we consider the degree to which the system output reflects the different aspects of 
- hurricane force wind warnings are in effect from Rhode Island Sound to Chincoteague Bay

- over 5000 commercial airline flights scheduled for October 28 and October 29 were cancelled

Figure 1: Example nuggets from Hurricane Sandy.

an event. Events are often composed of a variety of sub-events. For example, the Hurricane Sandy event includes sub-events related to the storm making landfall, the ensuing flooding, the many transportation issues, among many others. An ideal system would update the user about each of these sub-events as they occur. We refer to these sub-events as the nuggets associated with an event. A nugget is defined as a fine-grained atomic sub-event associated with an event. We present 2 example nuggets associated with the Hurricane Sandy event in Figure 1. Each event has anywhere from 50 to several hundred nuggets in total in our gold dataset. We describe how these nuggets are found in Section 4.

Throughout our treatment of our algorithm, the salience of an update captures the degree to which it reflects an event's unobserved nuggets. Assuming that we have a text representation for each of our nuggets, the salience of an update $u$ with respect to a set of nuggets $N$ is defined as,

$$
\text { salience }(u)=\max _{n \in N} \operatorname{sim}(u, n)
$$

where $\operatorname{sim}(\cdot)$ is the semantic similarity such as the cosine similarity of latent vectors associated with the update and nugget text (Guo and Diab, 2012).

\subsection{Update Summarization}

Our system architecture follows a simple pipeline design where each stage provides an additional level of processing or filtering of the input sentences. We begin with an empty update summary $U$. At each hour we receive a new batch of sentences $b_{t}$ from the stream of event relevant documents and perform the following actions:

1. predict the salience of sentences in $b_{t}$ (Section 3.2),

2. select a set of exemplar sentences in $b_{t}$ by combining clustering with salience predictions (Section 3.3),
3. add the most novel and salient exemplars to $U$ (Section 3.4).

The resultant list of updates $U$ is our summary of the event.

\subsection{Salience Prediction}

\subsubsection{Features}

We want our model to be predictive of sentence salience across different event instances so we avoid event-specific lexical features. Instead, we extract features such as language model scores, geographic relevance, and temporal relevance from each sentence.

Basic Features We employ several basic features that have been used previously in supervised models to rank sentence salience (Kupiec et al., 1995; Conroy et al., 2001). These include sentence length, the number of capitalized words normalized by sentence length, document position, and number of named entities. The data stream comprises text extracted from raw html documents; these features help to downweight sentences that are not content (e.g. web page titles, links to other content) or more heavily weight important sentences (e.g., that appear in prominent positions such as paragraph initial or article initial).

Query Features Query features measure the relationship between the sentence and the event query and type. These include the number of query words present in the sentence in addition to the number of event type synonyms, hypernyms, and hyponyms using WordNet (Miller, 1995). For example, for event type earthquake, we match sentence terms "quake", "temblor", "seism", and "aftershock".

Language Model Features Language models allow us to measure the likelihood of producing a sentence from a particular source. We consider two types of language model features. The first model is estimated from a corpus of generic news articles (we used the 1995-2010 Associated Press section of the Gigaword corpus (Graff and Cieri, 2003)). This model is intended to assess the general writing quality (grammaticality, word usage) of an input sentence and helps our model to select sentences written in the newswire style.

The second model is estimated from text specific to our event types. For each event type we create a corpus of related documents using pages and subcategories listed under a related 


\begin{tabular}{|lll|}
\hline Storm & Earthquake & Meteor Impact \\
Accident & Riot & Protest \\
Hostages & Shooting & Bombing \\
\hline
\end{tabular}

Figure 2: TREC TS event types.

Wikipedia category. For example, the language model for event type 'earthquake' is estimated from Wikipedia pages under the category Category:Earthquakes. Fig. 2 lists the event types found in our dataset. These models are intended to detect sentences similar to those appearing in summaries of other events in the same category (e.g. most earthquake summaries are likely to include higher probability for ngrams including the token 'magnitude'). While we focus our system on the language of news and disaster, we emphasize that the use of language modeling can be an effective feature for multi-document summarization for other domains that have related text corpora.

We use the SRILM toolkit (Stolcke and others, 2002) to implement a 5-gram Kneser-Ney model for both the background language model and the event specific language models. For each sentence we use the average token log probability under each model as a feature.

Geographic Relevance Features The disasters in our corpus are all phenomena that affect some part of the world. Where possible, we would like to capture a sentence's proximity to the event, i.e. when a sentence references a location, it should be close to the area of the disaster.

There are two challenges to using geographic features. First, we do not know where the event is, and second, most sentences do not contain references to a location. We address the first issue by extracting all locations from documents relevant to the event at the current hour and looking up their latitude and longitude using a publicly available geo-location service. Since the documents that are at least somewhat relevant to the event, we assume in aggregate the locations should give us a rough area of interest. The locations are clustered and we treat the resulting cluster centers as the event locations for the current time.

The second issue arises from the fact that the majority of sentences in our data do not contain explicit references to locations, i.e. a sequence of tokens tagged as location named entities. Our intuition is that geographic relevance is important in the disaster domain, and we would like to take ad- vantage of the sentences that do have location information present. To make up for this imbalance, we instead compute an overall location for the document and derive geographic features based on the document's proximity to the event in question. These features are assigned to all sentences in the document.

Our method of computing document-level geographic relevance features is as follows. Using the locations in each document, we compute the median distance to the nearest event location. Because document position is a good indicator of importance we also compute the distance of the first mentioned location to the nearest event location. All sentences in the document take as features these two distance calculations. Because some events can move, we also compute these distances to event locations from the previous hour.

Temporal Relevance Features As we track events over time, it is likely that the coverage of the event may die down, only to spike back up when there is a breaking development. Identifying terms that are "bursty," i.e. suddenly peaking in usage, can help to locate novel sentences that are part of the most recent reportage and have yet to fall into the background.

We compute the IDF for each hour in our data stream. For each sentence, the average TF*IDF for the current hour $t$ is taken as a feature. Additionally, we use the difference in average TF*IDF from time $t$ to $t-i$ for $i=\{1, \ldots, 24\}$ to measure how the TF*IDF scores for the sentence have changed over the last 24 hours, i.e. we keep the sentence term frequencies fixed and compute the difference in IDF. Large changes in IDF value indicate the sentence contains bursty terms. We also use the time (in hours) since the event started as a feature.

\subsubsection{Model}

Given our feature representation of the input sentences, we need only target salience values for model learning. For each event in our training data, we sample a set of sentences and each sentence's salience is computed according to Equation 1. This results in a training set of sentences, their feature representations, and their target salience values to predict.

We opt to use a Gaussian process (GP) regression model (Rasmussen and Williams, 2006) with a Radial Basis Function (RBF) kernel for the salience prediction task. Our features fall naturally 
into five groups and we use a separate RBF kernel for each, using the sum of each feature group RBF kernel as the final input to the GP model.

\subsection{Exemplar Selection}

Once we have predicted the salience for a batch of sentences, we must now select a set of update candidates, i.e. sentences that are both salient and representative of the current batch. To accomplish this, we combine the output of our salience prediction model with the affinity propagation algorithm. Affinity propagation (AP) is a clustering algorithm that identifies a subset of data points as exemplars and forms clusters by assigning the remaining points to one of the exemplars (Frey and Dueck, 2007). AP attempts to maximize the net similarity objective

$$
\mathcal{S}=\sum_{i: i \neq e_{i}}^{n} \operatorname{sim}\left(i, e_{i}\right)+\sum_{i: i=e_{i}}^{n} \text { salience }\left(e_{i}\right)
$$

where $e_{i}$ is the exemplar of the $i$-th data point, and functions sim and salience express the pairwise similarity of data points and our predicted apriori preference of a data point to be an exemplar respectively. AP differs from other $k$-centers algorithms in that it simultaneously considers all data points as exemplars, making it less prone to finding local optima as a result of poor initialization. Furthermore, the second term in $\mathcal{S}$ incorporates the individual importance of data points as candidate exemplars; most other clustering algorithms only make use of the first term, i.e. the pairwise similarities between data points.

AP has several useful properties and interpretations. Chiefly, the number of clusters $k$ is not a model hyper-parameter. Given that our task requires clustering many batches of streaming data, searching for an optimal $k$ would be computationally prohibitive. With AP, $k$ is determined by the similarities and preferences of the data. Generally lower preferences will result in fewer clusters.

Recall that salience $(s)$ is a prediction of the semantic similarity of $s$ to information about the event be summarized, i.e. the set of event nuggets. Intuitively, when maximizing objective function $\mathcal{S}$, AP must balance between best representing the input data and representing the most salient input. Additionally, when the level of input is high but the salience predictions are low, the preference term will guide AP toward a solution with fewer clusters; vice-versa when input is very salient on average but the volume of input is low. The adaptive nature of our model differentiates our method from most other update summarization systems.

\subsection{Update Selection}

The exemplar sentences from the exemplar selection stage are the most salient and representative of the input for the current hour. However, we need to reconcile these sentences with updates from the previous hour to ensure that the most salient and least redundant updates are selected. To ensure that only the most salient updates are selected we apply a minimum salience threshold; after exemplar sentences have been identified, any exemplars whose salience is less than $\lambda_{\text {sal }}$ are removed from consideration.

Next, to prevent adding updates that are redundant with previous output updates, we filter out exemplars that are too similar to previous updates. The exemplars are examined sequentially in order of decreasing salience and a similarity threshold is applied, where the exemplar is ignored if its maximum semantic similarity to any previous updates in the summary is greater than $\lambda_{\text {sim }}$.

Exemplars that pass these thresholds are selected as updates and added to the summary.

\section{Data}

For the document stream, we use the news portion of the 2014 TREC KBA Stream Corpus (Frank et al., 2012). The documents from this corpus come from hourly crawls of the web covering October 2011 through February 2013.

Our experiments also make use of the TREC Temporal Summarization (TS) Track data from 2013 and 2014 (Aslam et al., 2013). This data includes 25 events and event metadata (e.g., a user search query for the event, the event type, and event evaluation time frame). All events occurred during the time span of the TREC KBA Stream Corpus. For each event we create a stream of relevant documents by selecting only documents that contain the complete set of query words.

Along with the metadata, NIST assessors constructed a set of ground truth nuggets for each event. Nuggets are brief and important text snippets that represent sub-events that should be conveyed by an ideal update summary. In order to accomplish this, for each event, assessors were provided with the revision history of the Wikipedia page associated with the event. For example, the 
revision history for the Wikipedia page for 'Hurricane Sandy' will contain text additions including those related to individual nuggets. The assessment task involves reviewing the Wikipedia revisions in the evaluation time frame and marking the text additions capturing a new, unique nugget. More detail on this process can be found in the track description (Aslam et al., 2013).

\section{Experiments}

We evaluate our system on two metrics: ROUGE (Lin, 2004), an automatic summarization method and an evaluation of system expected gain and comprehensiveness-metrics adapted from the TREC TS track (Aslam et al., 2013).

\subsection{Training and Testing}

Of the 25 events in the TREC TS data, 24 are covered by the news portion of the TREC KBA Stream Corpus. From these 24, we set aside three events to use as a development set. All system salience and similarity threshold parameters are tuned on the development set to maximize ROUGE-2 F1 scores.

We train a salience model for each event using 1000 sentences randomly sampled from the event's document stream.

We perform a leave-one-out evaluation of each event. At test time, we predict a sentence's salience using the average predictions of the 23 other models.

\subsection{ROUGE Evaluation}

ROUGE measures the ngram overlap between a model summary and an automatically generated system summary. Model summaries for each event were constructed by concatenating the event's nuggets. Generally, ROUGE evaluation assumes both model and system summaries are of a bounded length. Since our systems are summarizing events over a span of two weeks time, the total length of our system output is much longer than the model. To address this, for each system/event pair, we sample with replacement 1000 random summaries of length less than or equal to the model summary (truncating the last sentence when neccessary). The final ROUGE scores for the system are the average scores from these 1000 samples.

Because we are interested in system performance over time, we also evaluate systems at 12 hour intervals using the same regime as above. The model summaries in this case are retrospective, and this evaluation reveals how quickly systems can cover information in the model.

\subsection{Expected Gain and Comprehensiveness}

NIST developed metrics for evaluating update summarization systems as part of the TREC TS track. We present results on two of these metrics, the expected gain and comprehensiveness.

Expected Gain We treat the event's nuggets as unique units of information. When a system adds an update to its summary, it is potentially adding some of this nugget information. It would be instructive to know how much unique and novel information each update is adding on average to the summary. To that end, we define

$$
\mathbb{E}[\text { Gain }]=\frac{\left|S_{n}\right|}{|S|}
$$

where $S$ is the set of system updates, $S_{n}$ is the set of nuggets contained in $S$, and $|\cdot|$ is the number of elements in the set. To compute the set $S_{n}$ we match each system update to 0 or more nuggets, where an update matches a nugget if their semantic similarity is above a threshold. $S_{n}$ results from the unique set of nuggets matched. Because an update can map to more than one nugget, it is possible to receive an expected gain greater than 1 . An expected gain of 1 would indicate that every sentence was both relevant and contained a unique piece of information.

Comprehensiveness Additionally, we can use the nuggets to measure the completeness of an update summary. We define

$$
\text { Comprehensiveness }=\frac{\left|S_{n}\right|}{|N|}
$$

where $N$ is the set of event nuggets. A comprehensiveness of 1 indicates that the summary has covered all nugget information for the event; the maximum attainable comprehensiveness is 1 .

Update-nugget matches are computed automatically; a match exists if the semantic similarity of the update/nugget pair is above a threshold. Determining an optimal threshold to count matches is difficult so we evaluate at threshold values ranging from .5 to 1 , where values closer to 1 are more conservative estimates of performance. A manual inspection of matches suggests that semantic similarity values around .7 produce reasonable matches. The average semantic similarity of 
manual matches performed by NIST assessors was much lower at approximately .25, increasing our confidence in the automatic matches in the .5-1 range.

\subsection{Model Comparisons}

We refer to our complete model as AP+SALIENCE. We compare this model against several variants and baselines intended to measure the contribution of different components. All thresholds for all runs are tuned on the development set.

Affinity Propagation only (AP) The purpose of this model is to directly measure the effect of integrating salience and clustering by providing a baseline that uses the identical clustering component, but without the salience information. In this model, input sentences are apriori equally likely to be exemplars; the salience values are uniformly set as the median value of the input similarity scores, as is commonly used in the AP literature (Frey and Dueck, 2007). After clustering a sentence batch, the exemplars are examined in order of increasing time since event start and selected as updates if their maximum similarity to the previous updates is less than $\lambda_{\text {sim }}$, as in the novelty filtering stage of AP+SALIENCE.

Hierarchichal Agglomerative Clustering (HAC) We provide another clustering baseline, single-linkage hierarchichal agglomerative clustering. We include this baseline to show that $\mathrm{AP}+\mathrm{SALIENCE}$ is not just an improvement over AP but centrality driven methods in general. HAC was chosen over other clustering approaches because the number of clusters is not an explicit hyper-parameter. To produce flat clusters from the hierarchical clustering, we flatten the HAC dendrogram using the cophenetic distance criteria, i.e. observations in each flat cluster have no greater a cophenetic distance than a threshold. Cluster centers are determined to be the sentence with highest cosine similariy to the flat cluster mean. Cluster centers are examined in time order and are added to the summary if their similarity to previous updates is below a similarity threshold $\lambda_{\text {sim }}$ as is done in the AP model.

Rank by Salience (RS) We also isolate the impact of our salience model in order to demonstrate that the fusion of clustering and salience prediction improves over predicting salience alone. In this model we predict the salience of sentences as in step 1 for AP+SALIENCE. We omit the cluster- ing phase (step 2). Updates are selected identically to step 3 of AP+SALIENCE, proceeding in order of decreasing salience, selecting updates that are above a salience threshold $\lambda_{\text {sal }}$ and below a similarity threshold $\lambda_{\text {sim }}$ with respect to the previously selected updates.

\section{Results}

\subsection{ROUGE}

\begin{tabular}{lccc}
\multicolumn{4}{c}{ ROUGE-1 } \\
\hline \hline System & Recall & Prec. & $\mathrm{F}_{1}$ \\
\hline AP+SALIENCE & $\mathbf{0 . 2 8 2}$ & $\mathbf{0 . 3 4 4}$ & $\mathbf{0 . 3 0 6}$ \\
AP & 0.245 & 0.285 & 0.263 \\
RS & 0.230 & 0.271 & 0.247 \\
HAC & 0.169 & 0.230 & 0.186 \\
\hline \multicolumn{4}{c}{} \\
\hline \hline Rystem & Recall & Prec. & $\mathrm{F}_{1}$ \\
\hline AP+SALIENCE & $\mathbf{0 . 0 4 5}$ & $\mathbf{0 . 0 5 6}$ & $\mathbf{0 . 0 4 9}$ \\
AP & 0.033 & 0.038 & 0.035 \\
RS & 0.031 & 0.037 & 0.034 \\
HAC & 0.017 & 0.024 & 0.019 \\
\hline
\end{tabular}

Table 1: System ROUGE performance.

Table 1 shows our results for system output samples against the full summary of nuggets using ROUGE. This improvement is statistically significant for all ngram precision, recall, and Fmeasures at the $\alpha=.01$ level using the Wilcoxon signed-rank test.

$\mathrm{AP}+\mathrm{SALIENCE}$ maintains its performance above the baselines over time as well. Figure 3 shows the ROUGE-1 scores over time. We show the difference in unigram precision (bigram precision is not shown but it follows similar curve). Within the initial days of the event, AP+SALIENCE is able to take the lead over the over systems in ngram precision. The AP+SALIENCE model is better able to find salient updates earlier on; for the disaster domain, this is an especially important quality of the model.

Moreover, the AP+SALIENCE's recall is not diminished by the high precision and remains competitive with AP. Over time AP+SALIENCE's recall also begins to pull away, while the other models start to suffer from topic drift.

\subsection{Expected Gain and Comprehensiveness}

Figure 4 shows the expected gain across a range of similarity thresholds, where thresholds closer 

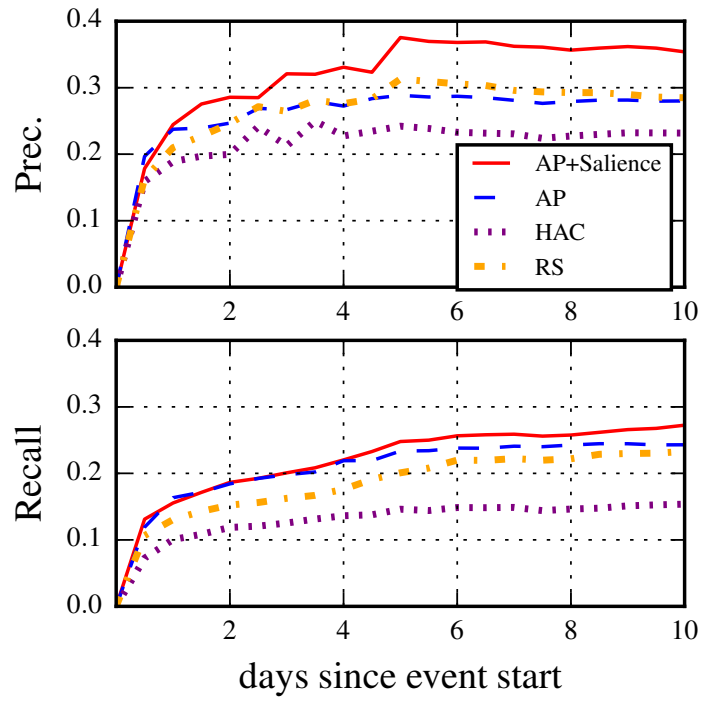

Figure 3: System ROUGE-1 performance over time.

to 1 are more conservative estimates. The ranking of the systems remains constant across the sweep with AP+S ALIENCE beating all baseline systems. Predicting salience in general is helpful for keeping a summary on topic as the RS approach out performs the clustering only approaches on expected gain.

When looking at the comprehensiveness of the summaries AP outperforms AP+S ALIENCE. The compromise encoded in the AP+SALIENCE objective function, between being representative and being salient, is seen clearly here where the performance of the AP+SALIENCE methods is lower bounded by the salience focused RS system and upper bounded by the clustering only AP system. Overall, AP+S ALIENCE achieves the best balance of these two metrics.

\subsection{Feature Ablation}

Table 2 shows the results of our feature ablation tests. Removing the language models yields a statistically significant drop in both ngram recall and F-measure. Interestingly, removing the basic features leads to an increase in both unigram and bigram precision; in the bigram case this is enough to cause a statistically significant increase in F-measure over the full model. In other words, the generic features actually lead to an inferior model when we can incorporate more appropriate domain specific features. The result mirrors Sparck Jones' claim that generic approaches to
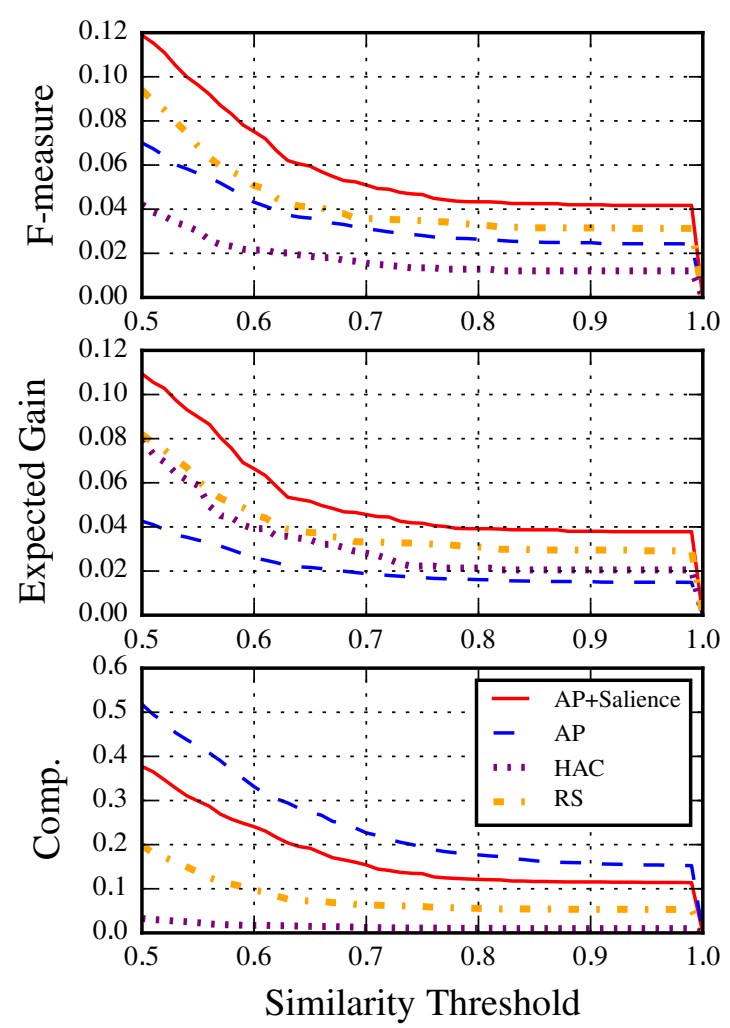

Figure 4: Expected Gain and Comprehensiveness performance.

summarization cannot produce a useful summary (Sparck-Jones, 1998).

Removing the language model and geographic relevance features leads to a statistically significant drop in ROUGE-1 F1 scores. Unfortunately, this is not the case for the temporal relevance features. We surmise that these features are too strongly correlated with each other, i.e. the differences in TF*IDF between hours are definitely not i.i.d. variables.

\section{Conclusion}

In this paper, we have presented an update summarization system for the disaster domain, and demonstrated improved system performance by integrating sentence salience with clustering.

We also have shown that features specifically targeted to the domain of disaster yield better summaries. We developed novel features that capture the language typical of different event types and that identify sentences specific to the particular disaster based on location.

In the future we would like to explore the appli- 
- $\quad$ The fire broke out when people in the building were trying to start their generator after the electricity went out.

- Pakistani television showed pictures of what appeared to be a three-story building with flames leaping from the top-floor windows and smoke billowing into the night sky.

- The people went to the back side of the building but there was no access, so we had to made forceful entries and rescue the people, said Numan Noor, a firefighter on the scene.

- "We have recovered 63 bodies, including three found when we reached the basement of the building," Karachi fire chief - $\quad$ Ehtesham Salim told AFP on Wednesday.

\section{Romanian Protests}

- Clashes between riot police and demonstrators have also erupted in the Romanian capital Bucharest for a third day in a row.

- $\quad$ BOC urged Romanians to understand that tough austerity measures are needed to avoid a default.

- More than 1,000 protesters rallied in Bucharest's main university square, blocking traffic.

- Bucharest : a Romanian medical official says 59 people suffered injuries as days of protests against the government and austerity measures turned violent.

Figure 5: AP+SALIENCE summary excerpts.

\begin{tabular}{lccc}
\multicolumn{4}{c}{ ROUGE-1 } \\
\hline \hline System & Recall & Prec. & $\mathrm{F}_{1}$ \\
\hline Full System & 0.282 & 0.344 & 0.306 \\
No Basic & 0.263 & $0.380^{\dagger}$ & 0.294 \\
No LM & $0.223^{\dagger}$ & 0.361 & $0.254^{\dagger}$ \\
No Time & $0.297^{\dagger}$ & $0.367^{\dagger \dagger}$ & $0.322^{\dagger}$ \\
No Geo & $0.232^{\dagger \dagger}$ & 0.381 & $0.265^{\dagger}$ \\
No Query & 0.251 & 0.377 & 0.280 \\
\hline
\end{tabular}

ROUGE-2

\begin{tabular}{lccc}
\hline \hline System & Recall & Prec. & $\mathrm{F}_{1}$ \\
\hline Full System & 0.045 & 0.056 & 0.049 \\
No Basic & 0.046 & $0.068^{\dagger \dagger}$ & $0.051^{\dagger}$ \\
No LM & $0.033^{\dagger}$ & 0.056 & $0.038^{\dagger}$ \\
No Time & $0.052^{\dagger \dagger}$ & $0.064^{\dagger \dagger}$ & $0.056^{\dagger \dagger}$ \\
No Geo & $0.037^{\dagger}$ & 0.065 & 0.042 \\
No Query & 0.043 & $0.068^{\dagger}$ & 0.048 \\
\hline
\end{tabular}

Table 2: Feature ablation ROUGE performance. $\dagger$ indicates statistically significant difference from full model at the $\alpha=.05$ level. $\dagger \dagger$ indicates statistically significant difference from full model at the $\alpha=.01$ level.

cation of the AP+SALIENCE model and features to a wider class of events.

\section{Acknowledgments}

The research described here was supported in part by the National Science Foundation (NSF) under IIS-1422863. Any opinions, findings and conclusions or recommendations expressed in this paper are those of the authors and do not necessarily re- flect the views of the NSF.

\section{References}

James Allan, Jaime G Carbonell, George Doddington, Jonathan Yamron, and Yiming Yang. 1998. Topic detection and tracking pilot study final report.

Javed Aslam, Matthew Ekstrand-Abueg, Virgil Pavlu, Fernado Diaz, and Tetsuya Sakai. 2013. Trec 2013 temporal summarization. In Proceedings of the 22nd Text Retrieval Conference (TREC), November.

James M Conroy, Judith D Schlesinger, PO Dianne, Mary E Okurowski, et al. 2001. Using hmm and logistic regression to generate extract summaries for duc.

Günes Erkan and Dragomir R Radev. 2004. Lexrank: Graph-based lexical centrality as salience in text summarization. J. Artif. Intell. Res.(JAIR), 22(1):457-479.

Elena Filatova and Vasileios Hatzivassiloglou. 2004. Event-based extractive summarization. In $A C L$ Workshop on Summarization, Barcelona, Spain.

John R Frank, Max Kleiman-Weiner, Daniel A Roberts, Feng Niu, Ce Zhang, Christopher Ré, and Ian Soboroff. 2012. Building an entity-centric stream filtering test collection for trec 2012. Technical report, DTIC Document.

Brendan J Frey and Delbert Dueck. 2007. Clustering by passing messages between data points. science, 315(5814):972-976.

David Graff and C Cieri. 2003. English gigaword corpus. Linguistic Data Consortium. 
Weiwei Guo and Mona Diab. 2012. A simple unsupervised latent semantics based approach for sentence similarity. In Proceedings of the First Joint Conference on Lexical and Computational SemanticsVolume 1: Proceedings of the main conference and the shared task, and Volume 2: Proceedings of the Sixth International Workshop on Semantic Evaluation, pages 586-590. Association for Computational Linguistics.

Qi Guo, Fernando Diaz, and Elad Yom-Tov. 2013. Updating users about time critical events. In Pavel Serdyukov, Pavel Braslavski, SergeiO. Kuznetsov, Jaap Kamps, Stefan Rüger, Eugene Agichtein, Ilya Segalovich, and Emine Yilmaz, editors, Advances in Information Retrieval, volume 7814 of Lecture Notes in Computer Science, pages 483-494. Springer Berlin Heidelberg.

Aria Haghighi and Lucy Vanderwende. 2009. Exploring content models for multi-document summarization. In Proceedings of Human Language Technologies: The 2009 Annual Conference of the North American Chapter of the Association for Computational Linguistics, pages 362-370. Association for Computational Linguistics.

Vasileios Hatzivassiloglou, Judith L Klavans, Melissa L Holcombe, Regina Barzilay, Min-Yen Kan, and Kathleen McKeown. 2001. Simfinder: A flexible clustering tool for summarization. Proceedings of the NAACL Workshop on Automatic Summarizatio.

Eduard Hovy and Chin-Yew Lin. 1998. Automated text summarization and the summarist system. In Proceedings of a workshop on held at Baltimore, Maryland: October 13-15, 1998, pages 197-214. Association for Computational Linguistics.

Julian Kupiec, Jan Pedersen, and Francine Chen. 1995. A trainable document summarizer. In Proceedings of the 18th annual international ACM SIGIR conference on Research and development in information retrieval, pages 68-73. ACM.

Hui Lin and Jeff Bilmes. 2011. A class of submodular functions for document summarization. In Proceedings of the 49th Annual Meeting of the Association for Computational Linguistics: Human Language Technologies-Volume 1, pages 510-520. Association for Computational Linguistics.

Chin-Yew Lin and Eduard Hovy. 2000. The automated acquisition of topic signatures for text summarization. In Proceedings of the 18th conference on Computational linguistics-Volume 1, pages 495-501. Association for Computational Linguistics.

Chin-Yew Lin. 2004. Rouge: A package for automatic evaluation of summaries. In Text Summarization Branches Out: Proceedings of the ACL-04 Workshop, pages 74-81.
George A Miller. 1995. Wordnet: a lexical database for english. Communications of the ACM, 38(11):39-41.

Ani Nenkova and Kathleen McKeown. 2012. A survey of text summarization techniques. In Mining Text Data, pages 43-76. Springer.

Ani Nenkova and Lucy Vanderwende. 2005. The impact of frequency on summarization. Microsoft Research, Redmond, Washington, Tech. Rep. MSR-TR2005-101.

Leysia Palen, Kenneth M Anderson, Gloria Mark, James Martin, Douglas Sicker, Martha Palmer, and Dirk Grunwald. 2010. A vision for technologymediated support for public participation \& assistance in mass emergencies \& disasters. In Proceedings of the 2010 ACM-BCS visions of computer science conference, page 8. British Computer Society.

Dragomir R Radev, Hongyan Jing, Małgorzata Styś, and Daniel Tam. 2004. Centroid-based summarization of multiple documents. Information Processing \& Management, 40(6):919-938.

Carl Edward Rasmussen and Christopher K. I. Williams. 2006. Gaussian Processes for Machine Learning. The MIT Press.

Karen Sparck-Jones. 1998. Automatic summarizing: factors and directions. In Advances in automatic text summarization, eds. Mani and Maybury.

Kate Starbird and Leysia Palen. 2013. Working and sustaining the virtual disaster desk. In Proceedings of the 2013 conference on Computer supported cooperative work, pages 491-502. ACM.

Andreas Stolcke et al. 2002. Srilm-an extensible language modeling toolkit. In INTERSPEECH.

Dingding Wang and Tao Li. 2010. Document update summarization using incremental hierarchical clustering. In Proceedings of the 19th ACM international conference on Information and knowledge management, CIKM'10, pages 279-288, New York, NY, USA. ACM.

William Yang Wang, Kapil Thadani, and Kathleen McKeown. 2011. Identifying event descriptions using co-training with online news summaries. In proceedings of IJCNLP, Chiang-Mai, Thailand, Nov. 